\title{
Estimation of model error for nonlinear system identification
}

\author{
V. Parameswaran \\ J.R. Raol
}

Indexing eerms: Algorithms, Model error, Nonlinear system identification

\begin{abstract}
Algorithms are presented for estimation of deterministic model error in the assumed models of nonlinear discrete and continuous time systenis. The explicit model error time histories are parameterised using least squares method. The parameterised models relative to the true model explain the deterministic deficiency in the chosen models, in the Sense of minimum model error. The algorithms have appealing features of extended Kalman filter. The numerical simulation results are obtained by implementing the algorithms in PC MATLAB.
\end{abstract}

\section{Introduction}

System identification and 'parameter estimation technology have reached a certain level of maturity, and there are many proven tools which can be routinely applied to model dynamic systems [I, 2]. The problem of determination of suitable structure and order of a system from its input/output data has several workable solutions, especially for linear systems [3]. Structure determination for nonlinear systems is relatively more complex. There are many real life situations for which accurate identification of nonlinear terms in the model of a dynamic system is required. One such example is related to the identification of aerodynamic model, based on Right data from high angle of attack (HAOA) manoeuvres [4]

In the present work, an approach to determine the discrepancy in the postulated model, hy the so-called minimum model error philosophy is used [5]. The method of invariant cmbedding is used to solve the resultant two-point boundary value (TPBV) problem, and to obtain very efficient and recursive algorithms to estimate the model error. The method of invariant embedding $[1,6]$ provides sequential estimates of the states of the nonlinear system. The estimates of deterministic model error, based on the measured data, are also obtained recursively in the present formulation. The time histories of these model discrepancy are then fitted using the least squares method to determine the coefficients of the model error description. Similarity and contradistinction of the estimators obtained by the present approach with that of extended Kalman filter are highlighted.
2 Model error estimation algorithms

\subsection{Discrete-timealgorithm}

Let the true nonlinear system be described as

$$
\begin{aligned}
& X(j+1)=g(X(j), j) \\
& Y(j)=h(X(h), j)
\end{aligned}
$$

Here $g$ is the true representation of the dynamic system with its states as $\mathrm{X} . Y$ is the vector of ohservahles obtained at discrete time intervals of $t_{0}<t_{j}<t_{N}$. The state and measurement vectors have appropriate dimensions. Eqns. 1 and 2 are recast to explicitly express the deterministic model error as follows [S]:

$$
\begin{aligned}
& x(j+1)=f(x(j), j)+d(j) \\
& y(j)=h(x(j), j)+v(j)
\end{aligned}
$$

Heref denotes the nominal model, the vector $v(j)$ denotes the random noise sequence with zero mean and covariance matrix $Q_{1}^{-1}$, and vector $d$ is the model error which is to he estimated in the sense of minimum model error criterion:

$$
\begin{aligned}
J= & \sum_{j=0}^{N}\left\{[y(j)-h(x(j), j)] Q_{1}[y(j)-h(x(j), j)]\right. \\
& \left.+d^{\prime}(j) Q_{2} d(j)\right\}
\end{aligned}
$$

Minimisation of $J$ obtains the condition of $x$ (the state of the model chosen to fit the data) tending very close to $X$ (the state of the true model or system). Thus the model discrepancy can he taken as the estimation of model error, the time histories of which can then be appropriately fitted in the sense of least squares to parameterise the model discrepancy. The minimisation of $\mathbf{J}$, by applying the Euler-Langrange conditions $[1,6]$ leads to the following TPBV (Appendix 6) problem:

$$
\begin{aligned}
x(j+1)= & f(x(j), j)+1 / 2 Q_{2}^{-1} \lambda(j) \\
\lambda(j-I)= & (\partial f(x(j), j) / \partial x)^{\prime} \lambda(j) \\
& +2(\partial h(x(j), j) / \partial x)^{\prime} Q_{1}[y(j)-h(x(j), j)]
\end{aligned}
$$

From eqns. 3 and 6 we get the expression for model discrepancy

$$
d(j)=1 / 2 Q_{2}^{-1} \lambda(j)
$$

The authors are grateful to Dr. S. Srinathkumar, Head, FMCD, NAL for his encouragement during the course of this work and lo the reviewers for their very useful comments and suggestions. 
The costate eqn. 7 is required to he solved to get the model correction terms ' $d$ ', The technique of invariant embedding is applied to solve the above TPBV problem [6]. The resultant set of estimator equations is given as:

$$
\begin{aligned}
& \begin{aligned}
& i(j+1)= f(\hat{x}(j), j)+2 S(j+1) H(j+1)^{\prime} \\
& \times Q_{1}[y(j+1)-h(\hat{x}(j+1), j+1)] \\
& S(j+1)=\left[I+2 P(j+1) H(j+1) Q_{1} H(j+1)\right] \\
& \times P(j+1) \\
& P(j+1)= f_{\dot{x}}(\hat{x}(j), j) S(j) f_{\hat{x}}(\hat{x}(j), j)^{\prime}+1 / 2 Q_{2}^{-1}
\end{aligned} \\
& \text { with } H(j)=\hat{c} h(x(j), j) / \hat{\partial} x(j) \text { at } x(j)=\hat{x}(j) \\
& \hat{d}(j)=2 S(j) H(j)^{\prime} Q_{1}[y(j)-h(\hat{x}(j), j)]
\end{aligned}
$$

Eqns. $9-12$ describe the invariant embedding based model error estimation (IEBMEE) algorithm in discrete time domain. Tuning of the IEBMEE algorithm is done by using appropriate values of matrices $Q_{1}$ and $Q_{2}[5,6]$. The algorithm presented above has structure similar to extended Kalman filtering algorithm. Since the system is deterministic, we interpret the inverse of matrix $S$ (and $\mathrm{P}$ ) as 'information providing matrix (IPM)'. Thus $\{P(j+1)\}^{-1}$ is a priori IPM and $\{S(j+1)\}^{-1}$ is the $a$ posteriori IPM, the latter taking into account the effect of measurements in improving the estimates. The weighting matrix $\boldsymbol{Q}$, affects the propagation of $\mathbf{S}$ which, in turn, aflects the estimator gain matrix $2 S H^{\prime} Q_{1}$. Thus both of the weighting matrices have significant roles to play in the convergence of the estimates to the true states. The matrix $Q$, governs the propagation of effect of errors in prediction of measurements, and matrix $Q$, governs the propagation of effect of errors in deterministic part of the model. This aspect has similarity with Kalman filtering algorithm: $Q_{2}^{-1}$ plays the role of covariance matrix of stochastic process noise. Thus the present algorithm based on the method of invariant embedding has some intuitively appealing features of extended Kalman filter and in contradistinction to it, obtains the estimation of deterministic model error in a sequential manner.

\subsection{Continuous-time algorithm}

The true nonlinear system is described as follows:

$$
\begin{aligned}
& \dot{X}(t)=g(X(t), t) \\
& Y(t)=H X(t)
\end{aligned}
$$

Here $g$ is the true representation of the dynamic system. The observables $Y$ are obtained for interval $t_{0}<I<\tau$. Following the development of the discrete-time case we get :

$$
\begin{aligned}
& \dot{x}(t)=f(x(t), t)+d(t) \\
& y(t)=H(t) x(t)+u(t)
\end{aligned}
$$

Here Jdenotes the nominal model, $v$ is additive measurement noise and the vector ' $d$ ' is the model discrepancy. The cost function is

$$
\begin{aligned}
J= & \int_{0}^{t}\left\{[y(t) \cdots H(t) x(t)]^{\prime} Q_{1}(t)[y(t)-H(t) x(t)]\right. \\
& \left.+d^{\prime}(t) Q_{2} d(t)\right\} d t
\end{aligned}
$$

Minimisation of $J[6]$ results in the TPBV problem:

$$
\begin{aligned}
& \dot{x}(t)=f(x(t), t)+1 / 2 Q_{2}{ }^{\prime}(t) \hat{\lambda}(t) \\
& \dot{\lambda}(t)=(-\hat{c} f(x(t), t) / \partial x)^{\prime} \lambda(t)
\end{aligned}
$$

$$
-2 H(t)^{\prime} Q_{1}(t)[y(t)-H(t) x(t)]
$$

$$
d(t)=1 / 2 Q_{2}^{-1}(t) \lambda(t)
$$

Application of invariant embedding to the TPBV problem results in

$$
\begin{aligned}
\hat{x}(t)= & f(\hat{x}(t), t)+2 S(t) H(t)^{\prime} Q_{1}(t)[y(t)-H(t) \hat{x}(t)] \\
\dot{S}(t)= & S(t) f_{\hat{x}}(\hat{x}(t), t)^{\prime}+f_{\hat{x}}(\hat{x}(t), t) S(t) \\
& -2 S(t) H(t)^{\prime} Q_{1}(t) H(t) S(t)+1 / 2 Q_{2}^{-1}(t) \\
d(t)= & 2 S(t) H(t)^{\prime} Q_{1}(t)[y(t)-H(t) \hat{x}(t)]
\end{aligned}
$$

Eqns. 21-23 describe the IEBMEE algorithm in continuous time. The inverse of matrix $S(t)$ can he interpreted as 'information providing matrix (IPM)'. Similar observations as made for discrete time case also apply here with appropriate interpretations. To solve the nonlinear matrix differential equation for $S$, the transformation $u=S z$, in eqn. 22 is used to obtain a set of linear differential equations [7]:

$$
\dot{S} z=S F^{\prime} z+F S z-2 S H^{\prime} Q_{1} H S z+1 / 2 Q_{2}^{-1} z
$$

or

$$
\dot{S} z+2 S H^{\prime} Q_{1} H S z-S F^{\prime} z=F u+1 / 2 Q_{2}^{-1} z
$$

Here $F=\partial / / \hat{\partial} x$; and since $u=\mathrm{Sz}$, we get $\dot{u}=\dot{S} z+S \dot{z}$ and $\dot{S}_{z}=\dot{u}-S \dot{z}$. Using Sz in eqn. 24 and defining $\dot{z}$ as in eqn. 25 we get :

$$
\begin{aligned}
& \dot{z}=-F^{\prime} z+2 H^{\prime} Q_{1} H u \\
& \dot{u}=1 / 2 Q_{2}{ }^{-1} z+F_{U}
\end{aligned}
$$

Eqns. 25 and 26 are solved by using transition matrix method.

\section{Numerical simulation results}

The simulation and estimation algorithms have been implemented using available and newly formulated $(m)$ functions in PC MATLAB. In the MATLAB implementation of the above equations the corresponding partial differentiation of the functions $f$ and $h$ is carried out by using finite difference method. This approach renders the computation of various quantities very straightforward for the above sequential equations. Thus any change of nonlinear model can be easily incorporated while iteratively scanning various nonlinear functions. The latter aspect offers great deal of flexibility in trying out various nonlinear functions/models for rea] data analysis.

\subsection{Discrete-time nonlinear system}

The true nonlinear model used has the following form:

$$
\begin{aligned}
X_{1}(j+1)= & 0.5 X_{1}(j)+0.623 X_{2}(j) \\
& -0.05 X_{1}^{3}(j)+2.5 \cos (0.3 j) \\
X_{2}(j+1)= & 0.1 X_{2}(j)+0.1 \cos (0.3 j)
\end{aligned}
$$

The model discrepancy is affected by eliminating one or more terms from eqn. 27. The deficient model is then used in the IEBMEE algorithm as ' $f$ '. In the first exercise of estimating the model error by the new algorithm, the cubic nonlinear term is ignored from the true model. The time history match of the true and estimated states $\left(x_{1}, x_{2}\right)$ is shown in Fig. 1. The comparison of the true and estimated model error time histories display excellent match. The corresponding model error time history is fitted with a general model of the form:

$$
d(j)=a_{1} X_{1}(j)+a_{2} X_{2}(j)+a_{3} X_{1}^{3}(j)+a_{4} X_{1}^{2}(j)
$$


It must be noted that square term is not present in the true model but it is intended to be estimated to see the performance of the algorithm. The estimates of the coeffi-
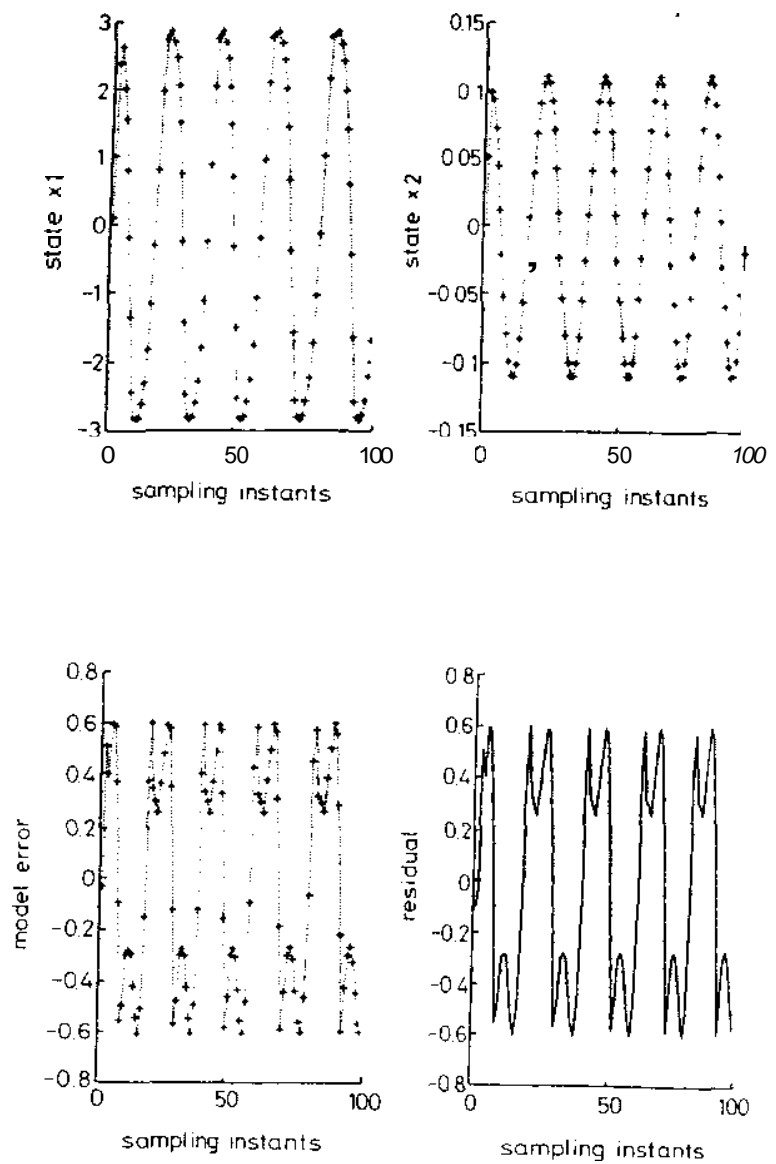

Fig. 1 Ti m histories of state, model error and residuals $\left(X_{1}, x_{1}^{3}\right.$ lerms d deted.DT wase II $)$

$--\rightarrow$ true

+ est.

cients of eqn. 29 are obtained by least squares method and are shown in Table 1. The number of data points used for state and parameter estimation are about 100 for

Table 1 : Nonlinear identification results: discrete-time

\begin{tabular}{llllll}
\hline Parameter & $\begin{array}{l}a, \\
\left(X_{1}\right)\end{array}$ & $\begin{array}{l}a_{2} \\
\left(X_{2}\right)\end{array}$ & $\begin{array}{l}a_{3} \\
\left(X_{3}^{3}\right)\end{array}$ & $\begin{array}{l}a_{4} \\
\left(X_{4}^{2}\right)\end{array}$ & $\begin{array}{l}\text { Terms } \\
\text { removed }\end{array}$ \\
\cline { 2 - 6 }$N$ & $(0.51$ & $(0.623)$ & $(-0.051$ & $(0)$ & - \\
I & $(0.5)$ & $(0.623)$ & -0.0499 & $\uparrow$ & $\boldsymbol{X}_{1}^{3}$ \\
$\mid 1$ & 0.4996 & $(0.623)$ & -0.05 & $\approx 1^{-4}$ & $X_{1}, X_{1}^{3}$ \\
111 & 0.4998 & 0.6161 & -0.05 & & $X_{1}, X_{2}, X_{1}^{3}$ \\
$V^{*}$ & 0.5000 & 0.6118 & -0.05 & $\downarrow$ & $X_{1}, X_{2}, X_{1}^{3}$ \\
\hline
\end{tabular}

Note: ( . ), $N=$ true values. ${ }^{*}$ Number of data points used $=6$.

cases I-III. The table also gives complete results of the system identification using the IEBMEE-LS algorithm when additional terms are deleted from the true model. Even when only six data points are used for estimation of model error and least squares fit, case IV, the results are quite accurate (Table 1, Fig. 2). The effect of initial conditjons of the states on the estimation accuracy was also studied. Even if a few initial data points of (estimated) states and the estimated model error are not used in the least squares parameter estimation process (when the initial guesses of the states are quite inaccurate), the coefficients are estimated with good accuracy. If the initial conditions are far away from the true (unknown) conditions, more initial data points would be required to be eliminated from the least squares process. This is not a limitation of the method.
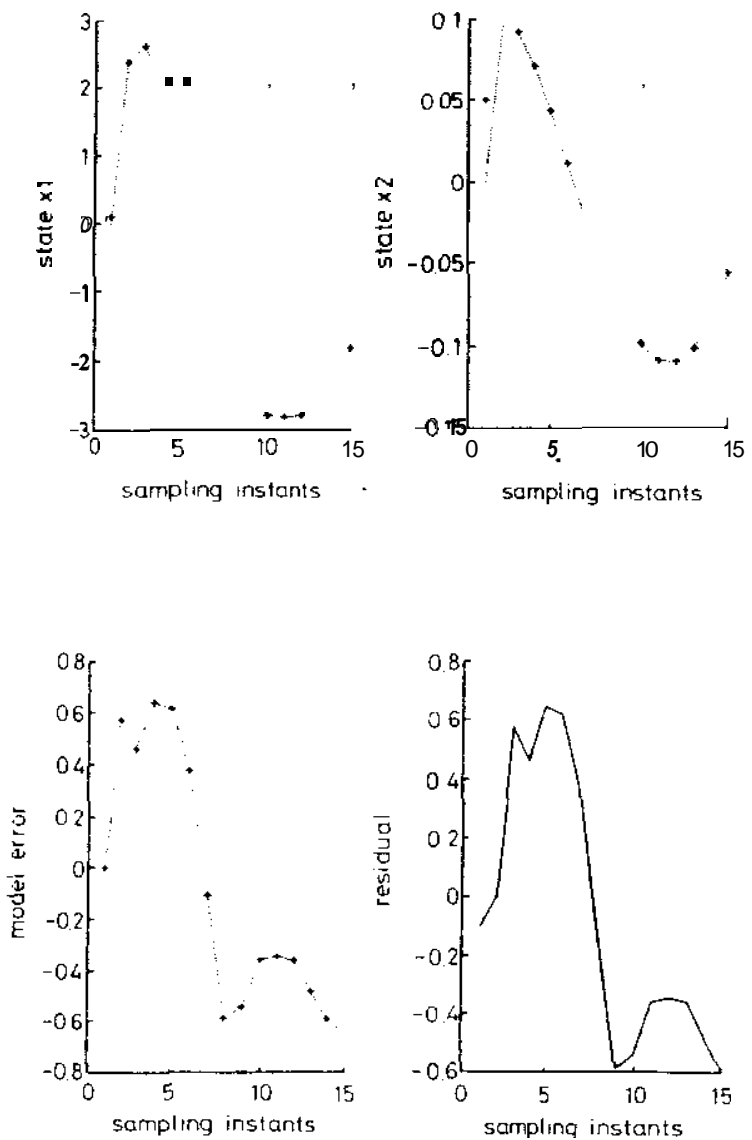

Fig. 2 Time histories of state, model error and residuals

$\left(X_{1}, X_{2}, X_{i}^{2}\right.$ (erms deleted-DT gase IV)

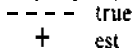

\subsection{Continuous-time nonlinear system}

The true nonlinear model used has the following form [5]:

$$
\dot{X}_{1}(t)=\left(2.5 \cos (t)-0.32 X_{1}(t)-X_{2}(t)-0.05 X_{2}^{3}(t)\right) / 2.56
$$

$$
\dot{X}_{2}(t)=X_{1}(t)
$$

Fig. 3 shows the results of estimation when two terms $\left(x_{2}\right.$ and $\left.x_{2}^{3}\right)$ are removed from the true model. The corresponding model error time history is fitted with a general model of the form:

$$
\begin{array}{r}
d_{1}\left(t_{j}\right)=a_{1} X_{1}\left(t_{j}\right)+a_{2} X_{2}\left(t_{j}\right)+a_{3} X_{2}^{3}\left(t_{j}\right)+a_{4} X_{2}^{2}\left(t_{j}\right) \\
j=1, \ldots, N
\end{array}
$$

The parameter estimation results are given in Table 2. The number of data points used for estimation are about 300 for cases I-Ill. The results of estimation using the IEBMEE-LS algorithm when other terms are deleted from the true model are given in Table 2. From the above two exercises it is clear that the IEB-MEE-LS algorithms correctly estimate the model error even for the cases where the models are made deficient by removing several terms and a few data points are used. The indicatio of the term which is not present is also very good the estimate ofa, being of the order of $10^{-4}$. If the initial values of 
the states are not very accurately known, one can iterate over the same equations, first solving them in forward direction and then, with the improved final states, in the
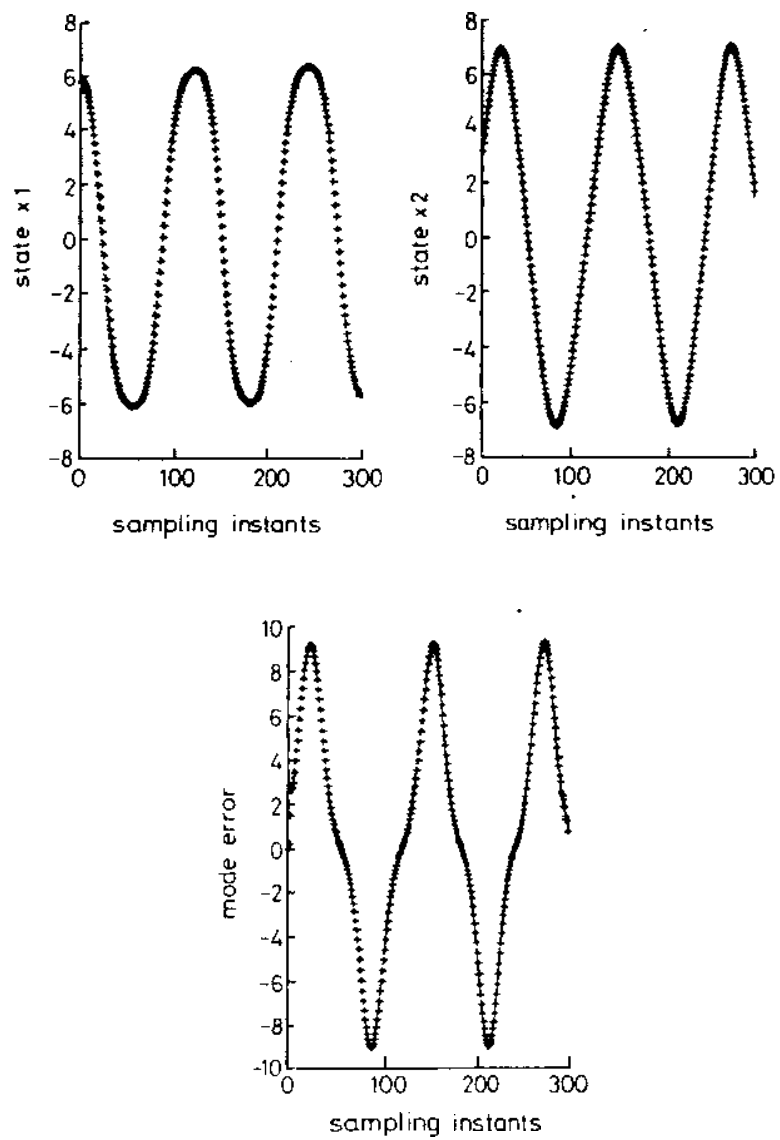

Fig. 3 Time histories of state and model error $\left(X_{2}, X_{2}^{3}\right.$ terms deleted-CT case II) --- true

+ est.

Table 2 : Nonlinear identification results: continuous-time

\begin{tabular}{|c|c|c|c|c|c|}
\hline Parameter & $\frac{-a}{\left(x_{1}\right)}$ & $\begin{array}{l}-a_{2} \\
\left(x_{2}\right)\end{array}$ & $\begin{array}{l}-a_{3} \\
\left(X_{2}^{3}\right)\end{array}$ & $\begin{array}{l}a_{4} \\
\left(X_{2}^{2}\right)\end{array}$ & $\begin{array}{l}\text { Terms } \\
\text { removed }\end{array}$ \\
\hline TV & $(0.125)$ & $(0.3906)$ & $(0.0195)$ & (0) & - \\
\hline I & $(0.125)$ & $(0.3906)$ & 0.0193 & & $x_{2}^{3}$ \\
\hline It & $(0.125)$ & 0.3931 & 0.0191 & $\approx 10^{-4}$ & $X_{2}, X_{2}^{3}$ \\
\hline IHI & 0.1242 & $(0.3906)$ & $(0.0195)$ & & $x_{1}$ \\
\hline IV* & $(0.125)$ & $(0.3906)$ & 0.0194 & $\downarrow$ & $X_{2}^{3}$ \\
\hline
\end{tabular}

${ }^{*}$ Less number of data used.

backward direction. This aspect retains the flexibility of sequential solution (estimates are updated at each sampling step) within an iterative process for further refinement of the estimates. However, for the case studies presented, such forward-backward iterations were not required.

\subsection{Aerodynamic parameter estimation}

It is important lo identify and estimate the contribution of nonlinear effects in an aerodynamic model of an aircraft from flight data obtained during HAOA dynamic manoeuvre. Light transport aircraft data were simulated for further validation of the algorithm presented in Section 2.2 using the true model:

$$
\begin{aligned}
\dot{w}= & \left(C_{z_{0}}+C_{z_{\alpha} \alpha} \alpha C_{z_{q}} q \bar{c} / U_{0}+C_{z_{\delta e}} \delta e\right) \bar{q} S / m \\
& +\mathrm{qu}+\mathrm{g} \cos \theta
\end{aligned}
$$

$$
\begin{aligned}
q= & \left(C_{m_{0}}+C_{m_{a}} \alpha+C_{m_{\alpha} 2} \alpha^{2}+C_{m_{q}} q \bar{c} / U_{0}+C_{m_{b e}} \delta e\right) \\
& \times \bar{q} S \bar{c} / I_{y} \\
\dot{u}= & \left(C_{x_{0}}+C_{x_{\alpha} \alpha} \alpha+C_{x_{\alpha} 2} \alpha^{2}\right) \bar{q} S / m-q w-g \sin \theta \\
0= & q
\end{aligned}
$$

Here $\boldsymbol{w}$ is the vertical velocity, $\mathrm{q}$ is the pitch rate, $\boldsymbol{u}$ is the forward component of aircraft velocity vector and $\theta$ is the pitch angle of the aircraft. The $\boldsymbol{C} \boldsymbol{s}$ represent various aerodynamic derivatives which are to be estimated. The true model contains nonlinear terms in $\mathrm{q}$ and $u$ equations. The deficient model is formulated by deleting the two nonlinear terms from the state eqns. 34 and 35. The simulated data were generated by using a doublet input, $\delta e$, as the elevator control surface deflection. The sampling time was $30 \mathrm{~ms}$. Fig. 4 shows the time histories of the true and
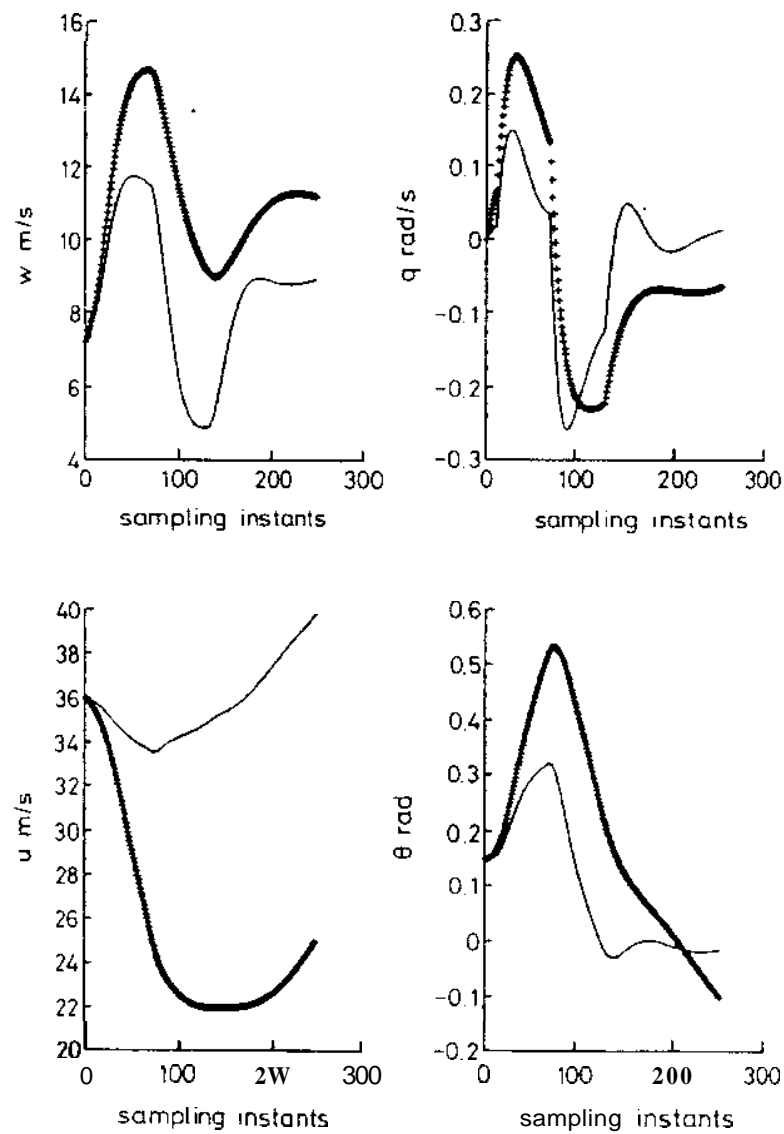

Fig. 4 Time histories of true and deficient states

$$
\begin{aligned}
& \left(C_{x^{2}} ; C_{m \alpha^{2}} \text { terms deleted+aircraft example }\right) \\
& +\quad \text { ine } \\
& \text { def. state }
\end{aligned}
$$

deficient states. Using the continuous-time IEBMEE algorithm, the states are estimated recursively. Fig. 5 shows the true and estimated states. To determine the functional dependence of the model error on the system states, $d(t)$ must be converted into explicit function of $x(t)$, given by $K(x(t), t)$. Once $\mathrm{K}$ is found, it is added to the deficient model ' $f$ ' to produce the true model of the system $(g=/+K)$. To determine the correct form of $\mathrm{K}$, for each element of $d$, the time history of a candidate function $\mathrm{K}$ is formed using the estimated states and is correlated with the model error estimates. The linear correlation coefficient $\rho$ for the pair $\left\{d_{i}(t), k_{i}(t)\right)$ is then computed [8]. When $|\rho(d, k)|=I, d(t)$ is linearly related to the candidate form. This form $k_{i}$ is parameterised to vield the final form $K$. The correlation results of the deficient model are given in Table 3 and the results of 


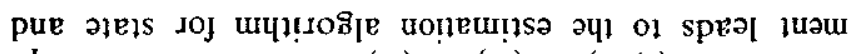

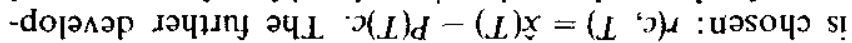
$0=\supset$ jo pooyınoquộว

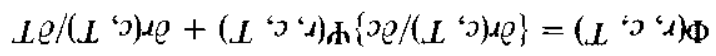

:se

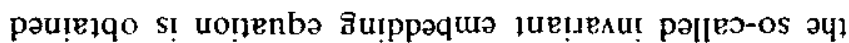

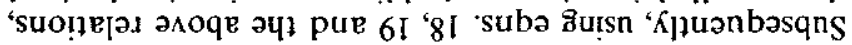

$$
(L \nabla+L \cdot \partial \nabla+\jmath) \mu=(L \nabla+L) x
$$

$$
\begin{aligned}
& x \nabla+(L \cdot) \mu= \\
& (L \nabla+L)^{x} \leftarrow \partial \nabla+\mathcal{\partial}=(L \nabla+L) \zeta \\
& (L \cdot O) \mu=(L) x \leftarrow)=(L) V
\end{aligned}
$$

: pəus!

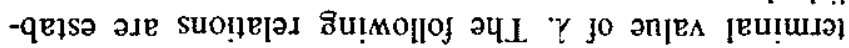

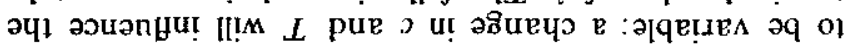

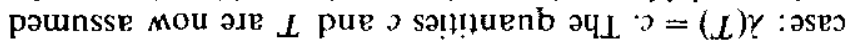

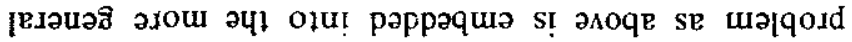

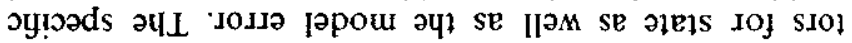

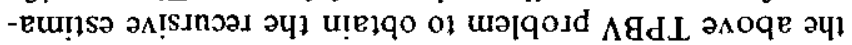

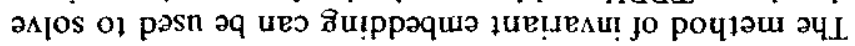

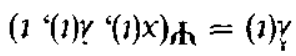

$$
\begin{aligned}
& (z(j) \nvdash(i) x) \Phi=(i) x
\end{aligned}
$$

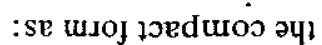

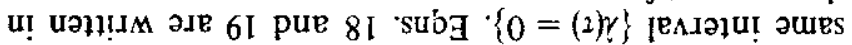

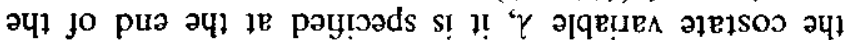

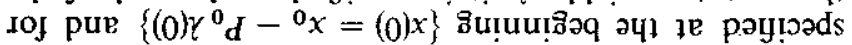

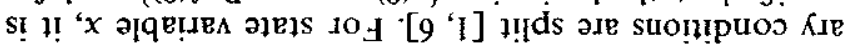

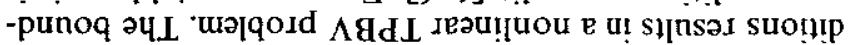

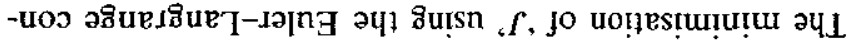

бu!ppəquә

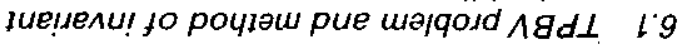

$$
\mathbf{x} \text { purdd } \mathbf{9}
$$

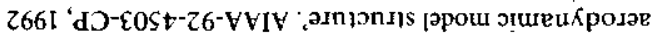

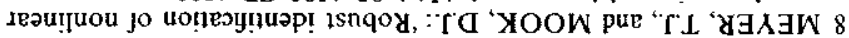

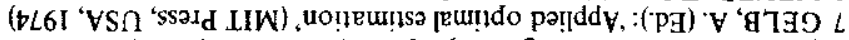

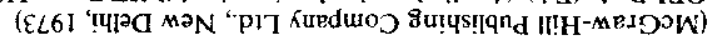

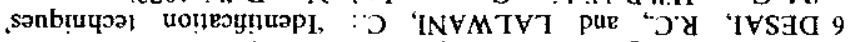

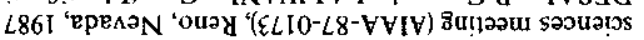

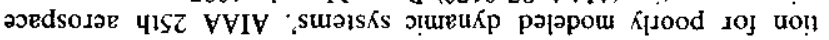

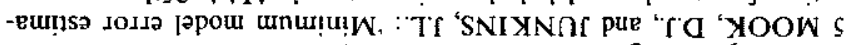
I86I' '9I6I dI VSVN" :uO!ssaläa as!mdals pay

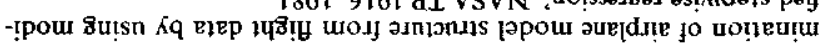

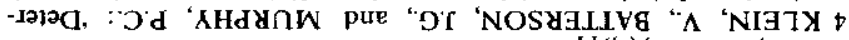
29-9S dd '(f) ' $\mathrm{ZI}-\mathrm{OWWS}$ ' $266 \mathrm{I}$

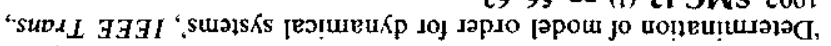

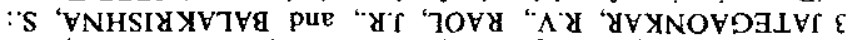

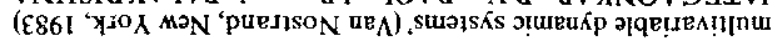

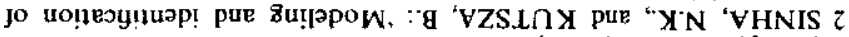
$(\rightarrow L 6 I$ ' $x$ J

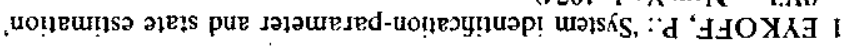

$$
\text { seoursajey }
$$

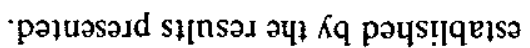

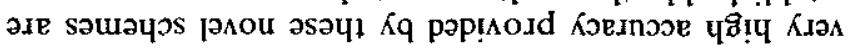

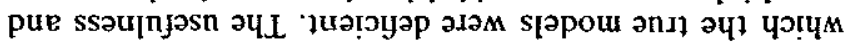

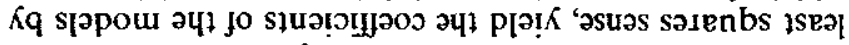

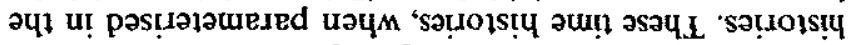

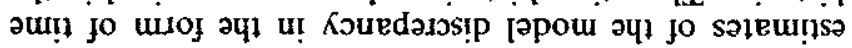

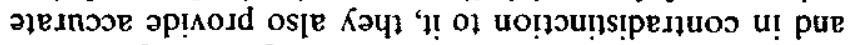

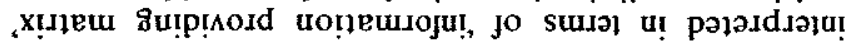

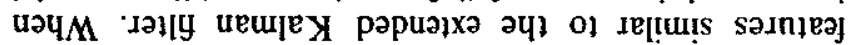

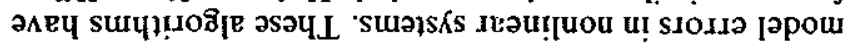

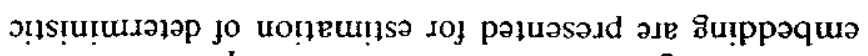

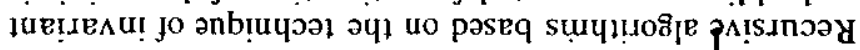

\begin{tabular}{|c|c|c|}
\hline 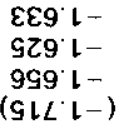 & $\begin{array}{c}299^{\circ} \varepsilon \\
6 \hookleftarrow 9^{\circ} \varepsilon \\
909^{\circ} \varepsilon \\
\left(609^{\circ} \varepsilon\right)\end{array}$ & $\begin{array}{r}\text { OL YNS } \\
\text { OZ YNS } \\
\text { əS!̣ ou ou } \\
\wedge\end{array}$ \\
\hline & & \\
\hline
\end{tabular}

$$
\text { suoisnjotios }
$$

\begin{tabular}{|c|c|c|c|}
\hline $\begin{array}{l}6966^{\circ} 0 \\
86 \angle 6^{\circ} 0 \\
\angle 866^{\circ} 0 \\
2866^{\circ} 0 \\
966^{\circ} 0 \\
8986^{\circ} 0 \\
9666^{\circ} 0\end{array}$ & 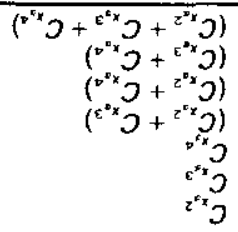 & $\begin{array}{l}0 \angle 66^{\circ} 0 \\
0086^{\circ} 0 \\
8866^{\circ} 0 \\
Z 866^{\circ} 0 \\
6696^{\circ} 0 \\
\nabla 986^{\circ} 0 \\
9666^{\circ} 0\end{array}$ & 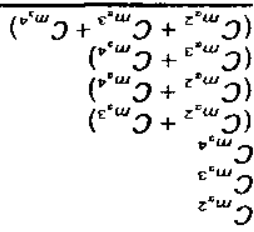 \\
\hline
\end{tabular}

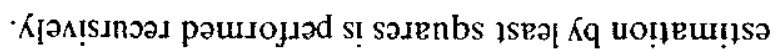

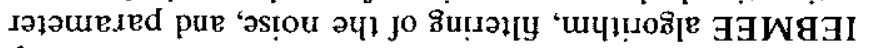

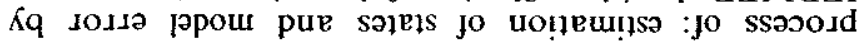

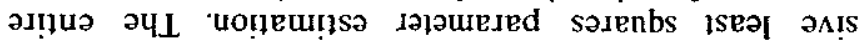

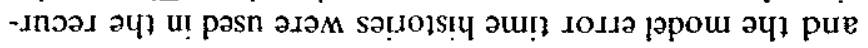

o!̣led as!ou ol |eu6! = HNS

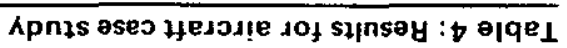

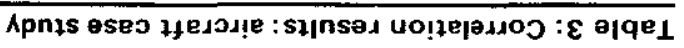

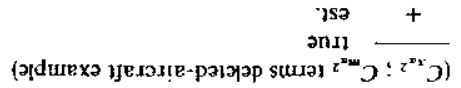
sajols palbuinsa pup and fo sa!sols!y au! $\quad \mathbf{9} \cdot \mathbf{6 ! d}$

s?udisu! bu!fduds

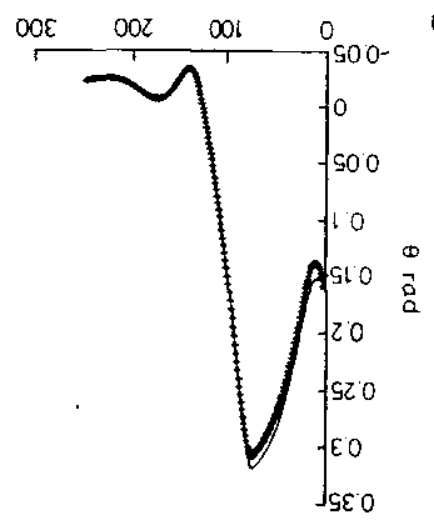

słudisu! Sulfduros

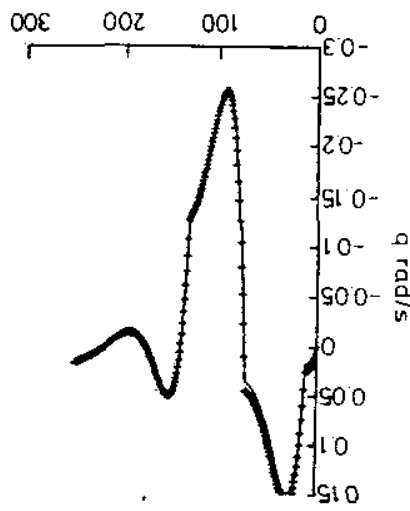

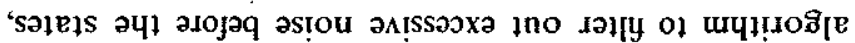

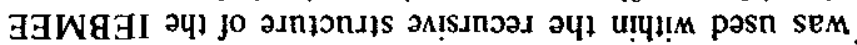

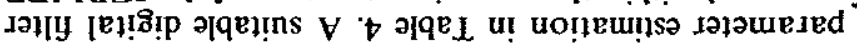


-model error (with $T$ considered as a running variable) as given in the text with

$$
x(0)=x_{0}-P_{0} \lambda(0) \quad \text { and } \hat{x}(0)=x_{0} ; P(0)=P_{0}
$$

Thus, since the terminal conditions are held invariant, the solution of the special case, the TPBV problem under question, is obtained from the solution of the more general problem. 